\title{
The Alchemical Energy Landscape for a Pentameric Cluster
}

John W. R. Morgan ${ }^{1, \text { a) }}$ and Sharon C. Glotzer ${ }^{2}$

1) University Chemical Laboratories, Lensfield Road, Cambridge CB2 $1 E W, U K^{\text {b) }}$

${ }^{2)}$ Department of Chemical Engineering, University of Michigan, Ann Arbor, USA ${ }^{\mathrm{c})}$

We investigate the energy landscape of an alchemical system of point particles in which the parameters of the interparticle potential are treated as degrees of freedom. Using geometrical optimization, we locate minima and transition states on the landscape for pentamers. We show that it is easy to find the parameters that give the lowest energy minimum, and that the distribution of minima on the alchemical landscape is concentrated in particular areas. In contrast to the usual changes to an energy landscape when adding more degrees of freedom, we find that introducing alchemical degrees of freedom can reduce the number of minima. Moreover, compared to landscapes of the same system with fixed parameters, these minima on the alchemical landscape are separated by high barriers. We classify transition states on the alchemical landscape by whether they become minima or remain transition states when the potential parameters are fixed at the stationary point value. We show that those that become minima have a significant alchemical component in the direction of the pathway, while those that remain as transition states can be characterised mainly in terms of atomic displacements.

\section{INTRODUCTION}

A fundamental challenge in colloidal science is posed by the vast array of possible colloids that can be made. ${ }^{1-4}$ The complexity and time required to physically manufacture designer particles means that is not possible to fully explore the parameter space. Computer simulations allow a greater throughput and there has been some success using the energy landscape approach ${ }^{5}$ to predict assembly pathways and structures for colloidal systems. ${ }^{6-8}$

Typically, a computational investigation involves defining an interaction potential energy function with a set of parameters to describe the system. This interaction potential defines the model. Simulations may be run at different values of the parameters to explore the behaviour or locate a region of parameter space supporting behaviour of interest. An alternative approach is to allow the parameters themselves to vary in situ. This technique has been termed digital alchemy, ${ }^{9-11}$ because it involves changing the nature of the particles modeled during a simulation. The model parameters become variables rather than fixed quantities and can be changed by step-taking algorithms in Monte Carlo simulations ${ }^{12}$ or evolved dynamically in molecular dynamics simulations ${ }^{13,14}$, in a thermodynamically consistent way. ${ }^{9}$ Previous work has mostly focused on changing the particle shape, but the approach can be applied much more generally. By exploring a landscape with such extended degrees of freedom, it is possible to locate the optimal parameters for a particular structure much more quickly and to identify features that favour a particular target. ${ }^{15}$

Following previous work ${ }^{9,13,14}$ we investigate the potential energy landscape (PEL) of the oscillating pair potential (OPP). ${ }^{16}$ Originally developed in the context of metal alloys, this potential displays interesting phase behaviour, includ-

\footnotetext{
a) Electronic mail: jwrm2@cam.ac.uk

b) Department of Chemical Engineering, University of Michigan, Ann Arbor, USA

c) Department of Materials Science and Engineering, University of Michigan, Ann Arbor, USA; Biointerfaces Institute, University of Michigan, Ann Arbor, USA,
}

ing an icosahedral quasicrystal. ${ }^{17}$ Here we exploit it as a toy potential ${ }^{14}$ with two adjustable parameters that displays many local minima in the alchemical landscape. We define the alchemical landscape to mean the PEL including both the particle Cartesian coordinates and the alchemical coordinates as variables. Since the PEL contains the complete description of the system, ${ }^{5}$ we seek to understand the effects of including alchemical degrees of freedom on the landscape. As proof of concept, we survey the alchemical landscape for small clusters of particles, locating energy minima and transition states, and compare with fixed-parameter landscapes. We find that introducing alchemical degrees of freedom can reduce the number of stationary points and increase barrier heights between them. We show that alchemical transition states fall into two classes: those that are minima and those that are transition states in the equivalent fixed landscape, and we calculate the alchemical contributions along the path for each class.

\section{METHODS}

\section{A. Oscillating Pair Potential}

The form of the OPP is ${ }^{16}$

$$
\begin{aligned}
V(\mathbf{r}) & =\sum_{i \neq j} V_{i j}\left(\mathbf{r}_{i}, \mathbf{r}_{j}\right), \\
V_{i j}\left(\mathbf{r}_{i}, \mathbf{r}_{j}\right) & =\frac{\varepsilon \sigma^{15}}{r_{i j}^{15}}+\frac{\varepsilon \sigma^{3}}{r_{i j}^{3}} \cos \left(k\left(\frac{r_{i j}}{\sigma}-1.25\right)-\phi\right),
\end{aligned}
$$

where $r_{i j}$ is the distance between any two particles and the sum runs over all pairs of particles. $V_{i j}\left(\mathbf{r}_{i}, \mathbf{r}_{j}\right)$ is the pair potential, $\varepsilon$ is the unit of energy and $\sigma$ is the unit of distance. The alchemical parameters are $k$ and $\phi . k$ represents a frequency and controls the spacing between wells: a higher value of $k$ means there are more wells in the potential. The parameter $k$ was restricted to the range $5<k<20$, with a harmonic constraint towards the allowed region outside this range 


$$
V_{k}(k)= \begin{cases}\varepsilon k_{\text {rep }}(k-5)^{2}, & \text { if } k<5 \\ \varepsilon k_{\text {rep }}(k-20)^{2}, & \text { if } k>20 \\ 0, & \text { otherwise }\end{cases}
$$

We found a value of $k_{\text {rep }}=10^{4}$ to be sufficient for the harmonic repulsion constant. Any minima or transition states located outside the acceptable $k$ range were ignored. $\phi$ represents a phase and is periodic over the range $0 \leq \phi<2 \pi$, so no constraints were necessary.

Usually, a cutoff is not required in the pair potential when modeling clusters. ${ }^{18}$ Here, however, the potential continues to oscillate even at very long range, so without a cutoff there are effectively an infinite number of wells and an infinite number of local potential energy minima. To avoid this unphysical scenario, a cutoff was introduced. The Stoddard-Ford procedure $^{19}$, used to impose a cutoff in other studies ${ }^{6}$ proved to be unsuitable, as the persistent oscillations could make the gradient far from zero at the cutoff distance, introducing very large changes to the potential. Instead the $\mathrm{XPLOR}^{20,21}$ smoothing function was used, with the smoothing beginning at $r_{i j}=2.5$ and the potential and gradient going smoothly to 0 at $r_{i j}=3$.

\section{B. Generating Databases}

To generate a database of minima and transition states, basin-hopping (BH) global optimization ${ }^{5,22-24}$ as implemented in GMIN ${ }^{25}$ was first run to locate the global potential energy minimum and other low-lying minima. In this algorithm, a transformation is applied to the potential energy surface, in which all points are quenched to a local minimum, using the limited memory Broyden-Fletcher-Goldfarb-Shanno local minimizer. ${ }^{26}$ The transformed surface is searched using a Metropolis Monte-Carlo scheme. ${ }^{12}$ The transformation removes barriers from the landscape without changing the position or energy of minima, allowing for efficient searching. ${ }^{24}$

Located minima were used as end points for transition state searches using the doubly nudged ${ }^{27-29}$ elastic band ${ }^{30-34}$ (DNEB) algorithm and eigenvector following ${ }^{35}$ (EF) as implemented in $\mathrm{OPTIM}^{36}$. From an initial interpolation between minima, DNEB places images connected with harmonic springs, forming a band across the landscape. The energy of the band is the sum of the energies for each image with the energies of the springs. A local optimization of the band produces a pathway that hopefully passes close to true transition states, as defined by the Murrell-Laidler theorem. ${ }^{37}$ The maxima on the DNEB path are chosen as candidates for optimization to transition states by EF. The two steepest-descent paths were followed in each direction from transition states, giving minimum-transition state-minimum connections. ${ }^{38}$

A PATHSAMPLE ${ }^{39}$ database was initialised from these connections and expanded using additional double-ended and single-ended ${ }^{35,40,41}$ transition state searches. The expansion was continued until no new minima were located in a day of running searches on 12 CPU's, except for the high density fixed landscape that was stopped after three weeks.
The resulting database constitutes a kinetic transition network, from which phenomenological rates and thermodynamic properties can be extracted. ${ }^{42}$ If the network is not complete, meaning not all minima and transition states have been located, there may be some minima that are not connected by any known pathway to all other minima. In that case, we can identify the largest group of minima connected to all others in the group, being the largest connected component in the language of graph theory. NetworkX was used for this identification. ${ }^{43}$ Using the harmonic superposition approach $^{5,44-50}$, the partition function as a function of temperature $Z(T)$ can be written as a sum over partition functions for each minimum $\alpha$ :

$$
Z(T)=\sum_{\alpha} Z_{\alpha}(T)
$$

The partition function for a minimum can be approximately evaluated as

$$
Z_{\alpha}(T)=\frac{n_{\alpha} e^{-\beta V_{\alpha}}}{\left(\beta h \bar{v}_{\alpha}\right)^{\kappa}},
$$

where $\beta=1 / k_{B} T, V_{\alpha}$ is the potential energy of minimum $\alpha$, $n_{\alpha}$ is the number of permutation-inversion isomers of minimum $\alpha,{ }^{51} \bar{v}_{\alpha}$ is the geometric mean of the vibrational normal mode frequencies and $\kappa$ is the number of vibrational degrees of freedom. 5

From here, the heat capacity can be calculated in the standard way as ${ }^{52}$

$$
C_{V}=\left(\frac{\partial E}{\partial T}\right)_{N, V}
$$

where $N$ is the number of particles, $V$ is the volume and the internal energy $E$ is

$$
E=k_{B} T^{2}\left(\frac{\partial \ln Z(T)}{\partial T}\right)_{N, V} .
$$

\section{Digital Alchemy}

During alchemical simulations, the potential parameters $k$ and $\phi$ are allowed to vary and are optimized in the same manner as particle coordinates. The $\mathrm{BH}$ and transition state search procedures do not depend on the nature of the variables being optimized, so no modifications were needed. The BH procedure requires the first derivatives of the pair potential with respect to the alchemical parameters, which are

$$
\begin{aligned}
& \frac{\partial V_{i j}}{\partial k}=-\varepsilon \sin \left(k\left(\frac{r_{i j}}{\sigma}-1\right)-\phi\right)\left(\frac{r_{i j}}{\sigma}-1\right) \frac{\sigma^{3}}{r_{i j}^{3}}, \\
& \frac{\partial V_{i j}}{\partial \phi}=\varepsilon \sin \left(k\left(\frac{r_{i j}}{\sigma}-1\right)-\phi\right) \frac{\sigma^{3}}{r_{i j}^{3}} .
\end{aligned}
$$

Normal mode analysis ${ }^{53}$ and some transition state search algorithms ${ }^{27-34}$ benefit from analytical second derivatives, 
which include the mixed derivatives with respect to position and potential parameters. These derivatives are also straightforward; the explicit results are omitted for brevity. As the potential is multiplied by the XPLOR smoothing function, the derivatives must also be multiplied appropriately by XPLOR and its derivatives.

The PEL does not depend on the temperature or the mass of the particles. Hence questions about what mass is associated with the alchemical degrees of freedom and whether their response to temperature is the same as for particle Cartesian degrees of freedom are avoided. For calculating free energies, however, these questions must be addressed. ${ }^{13,14}$ In this work, the main results do not depend on the choice of mass, but we also calculate heat capacities as a post-processing tool only. The mass relating to each coordinate enters the expression for the heat capacity through the normal modes frequencies $\bar{v}_{\alpha}$ in equation (4) Here, we arbitrarily use the same mass for the alchemical degrees of freedom as for the atomic coordinates. Therefore, care should be taken to not overinterpret the value of the frustration index.

\section{Frustration Index}

A frustration index can be used to quantify the amount of frustration present in a landscape, ${ }^{54,55}$ defined in terms of low energy minima separated by high barriers. Here we use a recently developed index based on the barrier heights between the global minimum and the other minima. ${ }^{56}$ The frustration index $\widetilde{f}(T)$ is

$$
\tilde{f}(T)=\sum_{\alpha \neq \mathrm{gmin}} \frac{p_{\alpha}^{\mathrm{eq}}(T)}{1-p_{\mathrm{gmin}}^{\mathrm{eq}}(T)}\left(\frac{V_{\alpha}^{\dagger}-V_{\mathrm{gmin}}}{V_{\alpha}-V_{\mathrm{gmin}}}\right),
$$

where the sum runs over all minima other than the global minimum, $p_{\alpha}^{\mathrm{eq}}$ is the temperature dependent equilibrium occupation probability of minimum $\alpha, V_{\alpha}$ is the potential energy of minimum $\alpha$, and $V_{\alpha}^{\dagger}$ is the potential energy of the highest transition state on the lowest energy path between minimum $\alpha$ and the global minimum. To facilitate comparison between different systems, the temperature was scaled for each system by $T_{m}$, the value at which the heat capacity has a maximum for that system. The term in the probabilities contributes significantly only for low energy minima, while the term in the potentials compares the barrier separating the minimum $\alpha$ from the global minimum with the energy difference between $\alpha$ and the global minimum, so contributes significantly only for minima separated from the global minimum by high barriers. Thus the frustration index is large when there are many low energy minima with high barriers to the global minimum and is small otherwise.
TABLE I. $k$ and $\phi$ values for selected databases, with the number of minima and transition states in those databases, the number of minima in the largest connected component, and the energy of the lowest minimum found. The reason for the selection of these $k$ and $\phi$ values is summarised in the final column.

\begin{tabular}{ccccccc}
$k$ & $\phi$ & $N_{\min }$ & $N_{\mathrm{ts}}$ & $N_{\mathrm{con}}$ & $E_{\min } / \varepsilon$ & description \\
\hline variable & 6760 & 10386 & 2492 & -4.9693 & alchemical \\
6.00 & 1.00 & 61 & 714 & 58 & -1.9792 & low density \\
8.74 & 4.37 & 2931 & 12691 & 1184 & -4.9693 & global minimum \\
19.75 & 5.00 & 458977 & 489497 & 221179 & -4.6385 & high density
\end{tabular}

\section{RESULTS}

\section{A. Oscillating Pair Potential}

We sought the smallest cluster of particles that would display non-trivial behaviour when the alchemical landscape is explored. A tetramer displays only trivial behaviour, as all particles can be nearest neighbors in a regular tetrahedral arrangement. In the alchemical ensemble, the alchemical parameters adjust to make the nearest neighbor well as deep as possible. We found only 13 minima, corresponding to the $k$ and $\phi$ combinations that give a minimum in the first well depth with respect to changing $k$ and $\phi$.

In a pentamer, the five particles cannot all be nearest neighbors. Non-nearest neighbor interactions can still be optimized when they are at a distance corresponding to a well in the OPP potential different from the nearest-neighbor well. With alchemical variation of $k$ and $\phi$, these potential parameters can also adjust to make a particular distance more favourable, leading to more complicated behaviour than for the tetramer.

A database of minima and transition states was created for the five-particle cluster, including the alchemical degrees of freedom. Table I shows the size of the database and the energy of the lowest minimum found.

The low-lying minima on the alchemical landscape indicate both the most stable structures available to the system and the potential parameters required to make them as stable as possible. Unlike for the tetramer, the alchemical parameters for the global minimum are not simply those parameters that give the deepest first well in the potential, due to the competition between the nearest-neighbor and more distant interactions.

The database is not complete, as evidenced by the absence of pathways between all pairs of minima, but the slow rate of discovering new minima and transition states suggests that the majority of structures have been located. In fact, the only pathway between two arbitrary minima may consist of transition states and intermediate minima that lie outside the permitted $k$ range, so even with a complete database, the network may not be fully connected.

The distribution of minima throughout $k$ and $\phi$ space is shown in figure 1 . It is striking that most minima occur within a small region of alchemical space, with most combinations of parameters having no minima at all. Most minima occur around the $\phi$ value that gives the deepest nearest-neighbor well and at higher $k$ values. At high $k$, there are more wells in the potential and more possible distances between pairs of 


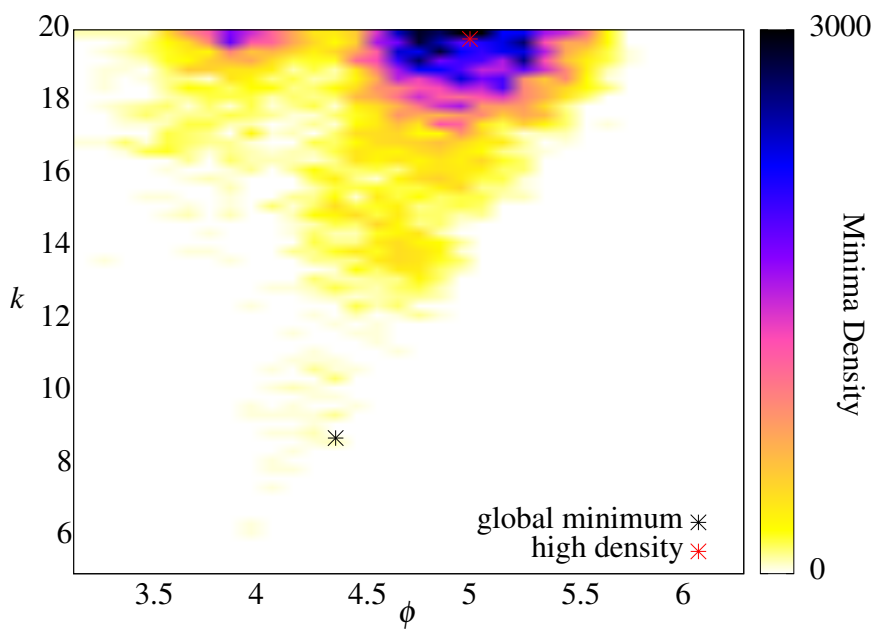

FIG. 1. The density of minima on the alchemical PEL as a function of the alchemical parameters $k$ and $\phi$. The region $0 \leq \phi<\pi$ is not shown as it contains no minima. Unit density corresponds to one minimum in an area of one $k$ unit by one $\phi$ unit. The points chosen for generating fixed parameter databases are also shown, except the low density point, which is not in the displayed region.

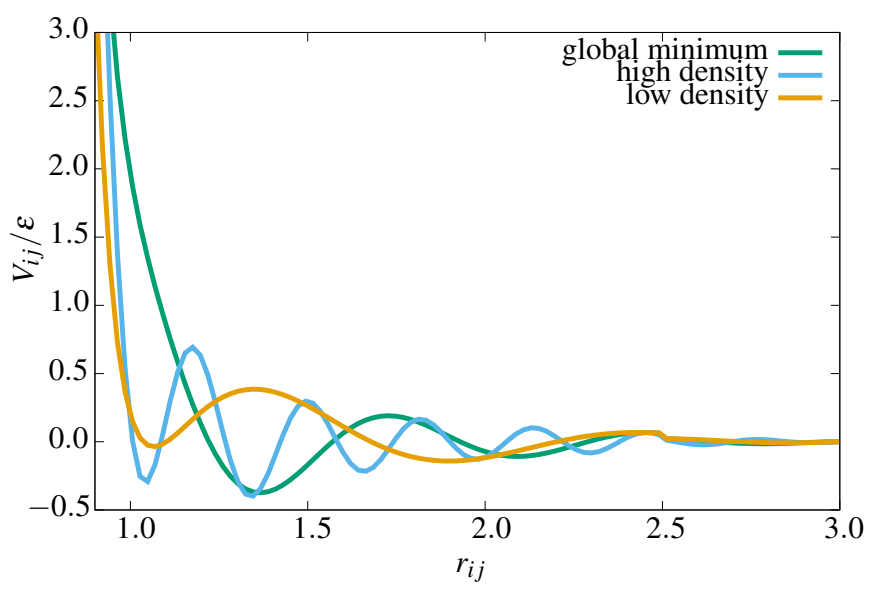

FIG. 2. The OPP potential for the three sets of fixed parameters used, as listed in table I.

particles, so it is not surprising that there are many more minima in this region.

Databases of minima and transition states at fixed alchemical parameters were also generated for comparison. A landscape with fixed alchemical parameters is a slice through the full alchemical landscape. Their parameters and characteristics are summarised in table I, with plots of the potential in figure 2. Three points on the alchemical landscape were selected, corresponding to the global minimum from the alchemical database, a region with a low density of minima on the alchemical landscape, and a region with a high density of minima on the alchemical landscape. In all cases, the global minimum five-particle cluster shape is a trigonal bipyramid (point group $D_{3 h}$ ), differing only in the distances between particles, which adjust to match the distance for the deepest well in the potential.
The fixed parameter database in the low density region is expectedly small, with no alchemical minima at these parameter values. In the fixed landscape at high alchemical minima density, the number of minima is much larger than the total number of alchemical minima. Allowing alchemical variation can reduce the number of minima and transition states on the landscape, as most structures that are stationary points with fixed parameters are no longer stationary points when they are allowed to vary.

This result stands in contrast to the usual changes to the topography of a PEL when adding more degrees of freedom by adding more particles: that the number of minima tends to increase exponentially with increasing numbers of particles. ${ }^{47,57,58}$ Most minima at fixed parameter values will not be minima in the alchemical space, as changes to the potential can usually be made that decrease the energy. Conversely, on adding a new particle to a system, minima will often exist with very similar interparticle distances to cluster structures before the addition. The main difference, then, is that changing the alchemical parameters affects all the pair distances together.

The landscapes for the four pentamer clusters are summarised in the disconnectivity graphs shown in figure $3.59,60$ Apart from the very different numbers of minima, the structures of the graphs are fairly similar, corresponding to "palm tree" funneled landscapes. ${ }^{60}$ The alchemical system graph is most similar to the fixed system in the region of high density of alchemical minima. Taken in conjunction with the density distribution in figure 1, it is clear that the contributions from the high density region dominate alchemical space.

To compare the landscapes more quantitatively, we calculated the frustration indices for the four systems, shown in figure 4. The alchemical landscape is very frustrated at low temperatures, indicating relatively high barriers between the global minimum and other minima with significant occupation probabilities. The energies and alchemical parameters of the ten lowest energy alchemical minima are shown in table II, demonstrating that the low energy minima are widely separated in alchemical space. The pathways between these minima cross unfavourable alchemical regions, giving high barriers. The other three systems exhibit lower frustration. At high temperatures, the frustration disappears for the alchemical system as the barriers become thermally accessible. Due to the higher frustration, we expect self-assembly on this alchemical landscape, in cases where it is possible to change the potential by altering experimental conditions, to be more difficult than on the fixed parameter landscapes, especially at low temperature. The system would become trapped in local minima with thermally inaccessible barriers preventing progress towards the global minimum.

All stationary points on the alchemical landscape must be stationary points on the corresponding fixed parameter landscape when the parameters are equal to those of the alchemical minimum. The inverse is not necessarily true: a stationary point on a fixed landscape is unlikely to remain a stationary point when the parameters are allowed to vary. An alchemical minimum must be a fixed parameter minimum, but an alchemical transition state could remain a transition state or become 

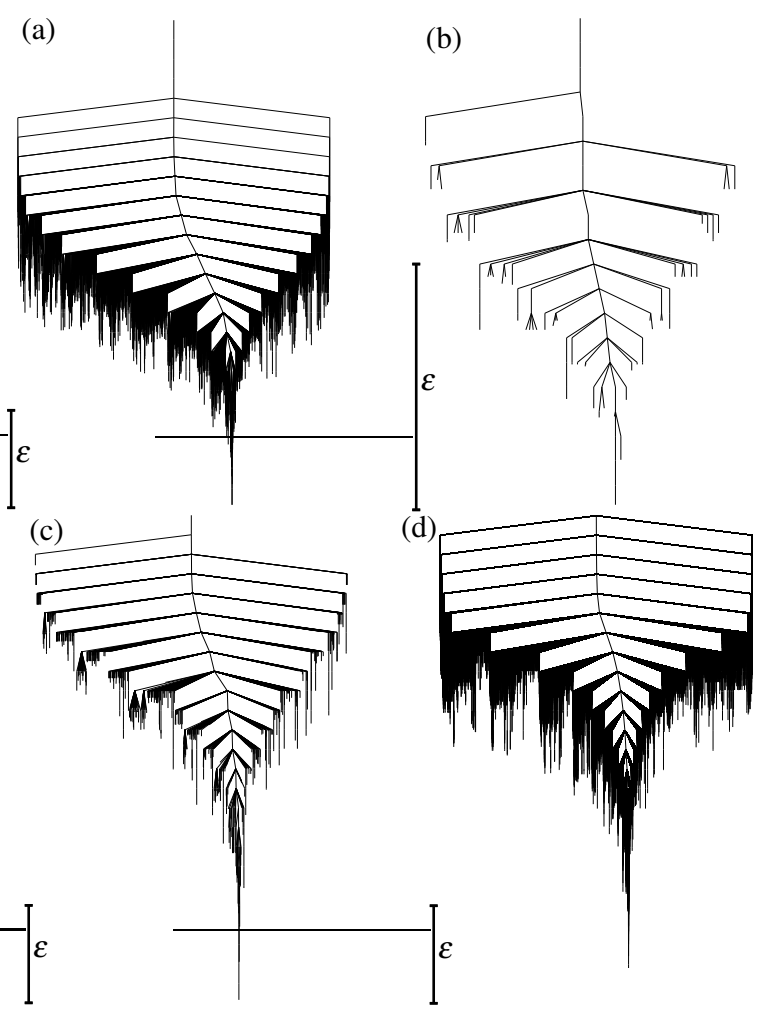

FIG. 3. Disconnectivity graphs for the alchemical landscape and the three fixed parameter landscapes, as listed in table I, showing the connected component including the global minimum. (a) The alchemical system. (b) The low density system. (c) The global minimum system. (d) The high density system.

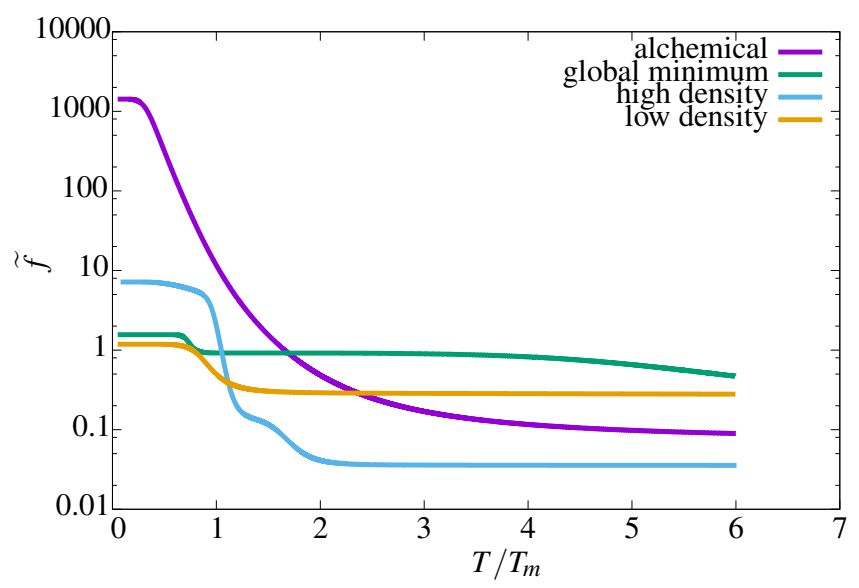

FIG. 4. The variation of the frustration indices with temperature for the four databases. The temperature has been scaled by the temperature of the greatest value of the heat capacity, $T_{m}$, for each database.

a minimum once the parameters are fixed. The second case indicates that the reaction coordinate at the transition state has significant contributions from the alchemical degrees of freedom. This situation is reminiscent of a martensitic transition, in which the reaction coordinate involves a change in lattice parameters rather than particle coordinates. ${ }^{61}$

Of the 10386 alchemical transition states in the database,
TABLE II. The energies, $k$ and $\phi$ values of the ten lowest energy alchemical minima. The minima are widely separated on the alchemical landscape.

\begin{tabular}{ccc} 
Energy $/ \varepsilon$ & $k$ & $\phi$ \\
\hline-4.9693 & 8.74 & 4.37 \\
-4.9690 & 17.42 & 5.60 \\
-4.7570 & 13.40 & 4.96 \\
-4.6784 & 15.38 & 5.24 \\
-4.5811 & 8.57 & 4.33 \\
-4.5798 & 17.02 & 5.50 \\
-4.5518 & 15.45 & 5.14 \\
-4.4844 & 17.43 & 5.40 \\
-4.4436 & 16.20 & 5.26 \\
-4.4314 & 18.19 & 5.32
\end{tabular}

TABLE III. RMS $k$ and $\phi$ contributions to the reaction coordinate at alchemical transition states, for those transition states that become minima when the parameters are fixed, and for those that remain transition states.

\begin{tabular}{ccc} 
Transition state becomes... & $k$ contribution & $\phi$ contribution \\
\hline Transition state & 0.0396 & 0.019 \\
Minimum & 0.497 & 0.154
\end{tabular}

346 became minima when the alchemical parameters were fixed. Although it may seem sensible to call these 'pure alchemical transitions', the reaction coordinate may not be precisely parallel to the alchemical degrees of freedom. We can quantify the 'alchemical content' of a transition state by taking the dot product of the reaction coordinate with a normalised vector parallel to the alchemical degrees of freedom. The reaction coordinate is the normalised eigenvector corresponding to the single negative Hessian eigenvalue at the transition state. ${ }^{37}$ In table III we present the root mean square (RMS) $k$ and $\phi$ content for both classes of alchemical transition state. While it is clear that those alchemical transition states that become minima tend to have a much greater alchemical component in the reaction coordinate, and that $k$ tends to have a greater contribution than $\phi$, neither class of transition is purely alchemical. Since any change in $k$ or $\phi$ changes the interparticle distances of the wells in the potential, such a change will always result in an adjustment of the particle Cartesian coordinates and a pure alchemical transition is not possible for this potential. Conversely, those transition states that remain as transition states tend to have a very small alchemical component and are almost purely described in terms of Cartesian coordinate displacements.

\section{B. Other Potentials}

In this contribution, we have not considered other potentials in detail. For simpler potentials, such as the Morse potential, ${ }^{62}$ intuition alone suffices. If the range parameter of the Morse potential were to be treated as an alchemical coordinate, then it would reduce towards zero (long range) so that all pair interactions would become as attractive as possible. Some form of normalisation would be required to avoid this. The most 
obvious option would be to scale the potential by the attractive area under the curve. However, intuition again indicates that with this approach, the range parameter would increase forever (short range), so that contribution from nearest neighbours would become as negative as possible. Instead, some other correction, based on reducing the depth of the well as the range increases, would be required. The details of this correction would have a strong influence on the alchemical landscape. These complications mean we do not envisage the alchemical landscape approach being of great utility for very simple potentials. Instead, we believe the approach will be useful for more complicated potentials with many tunable parameters, both potential parameters such as in the OPP potential and geometries of rigid bodies in coarse-grained systems. A search in alchemical space can be much more efficient than multiple searches at different values of parameters and can aid in choosing appropriate parameters. There are also applications in biological areas, with mutations between amino acids or RNA bases taking on the role of an alchemical parameters. As each use case will have specific challenges, such a study is beyond the scope of the current contribution.

\section{CONCLUSIONS}

We have explored the potential energy landscape of small clusters in an extended system where the parameters of the potential are also variables. As proof of concept, we have shown that geometrical approaches for exploring the landscape of such clusters are feasible and allow rapid location of the global minimum.

We compared some properties of the alchemical system to systems with fixed parameters, showing that there is a significant difference in the topographical change in the landscape upon introducing alchemical degrees of freedom compared to adding another particle. Although for small clusters with the OPP potential, it seems that high frustration on the alchemical landscape would hinder self-assembly in an experimental system, there is no difficulty for basin-hopping global optimization and theoretical prediction of the best parameters for self-assembly is possible.

We also analysed characteristics of pathways on the alchemical surface, showing that some are almost purely describable in terms of atomic displacements, while others contain a significant amount of alchemical character.

We expect that digital alchemy will become an important theoretical tool, as it has general applicability to a wide range of systems. In addition to ongoing work on optimizing colloidal shape and coarse-grained potentials with variable parameters, we envision 'floppy-bodies' - previously rigid bodies allowed to change shape in a restricted manner - and protein mutations.

\section{Conflicts of Interest}

There are no conflicts of interest to declare.

\section{ACKNOWLEDGMENTS}

The authors thank James Proctor, Pengji Zhou and David Wales for comments on the original manuscript. This work was supported in part by a grant from the Simons Foundation (256297, SCG) and also by the United Kingdom Engineering and Physical Sciences Research Council [grant number EP/N035003/1] (JWRM). Data may be accessed at <http://doi.org/10.5281/zenodo.3256234>

${ }^{1}$ Z. Zhang and S. C. Glotzer, "Self-assembly of patchy particles," Nano Lett. 4, 1407-1413 (2004).

${ }^{2}$ S. C. Glotzer and M. J. Solomon, "Anisotropy of building blocks and their assembly into complex structures," Nat. Mater. 6, 557-562 (2007).

${ }^{3}$ A. B. Pawar and I. Kretzschmar, "Fabrication, assembly, and application of patchy particles," Macromol. Rapid Commun. 31, 150-168 (2007).

${ }^{4}$ S. Sacanna and D. J. Pine, "Shape-anisotropic colloids: Building blocks for complex assemblies," Curr. Opin. Colloid Interface Sci. 16, 96-105 (2011).

${ }^{5}$ D. J. Wales, Energy landscapes (Cambridge University Press, 2003)

${ }^{6}$ J. W. R. Morgan, D. Chakrabarti, N. Dorsaz, and D. J. Wales, "Designing a Bernal spiral from patchy colloids," ACS Nano 7, 1246-1256 (2013).

${ }^{7}$ D. Morphew and D. Chakrabarti, "Supracolloidal reconfigurable polyhedra via hierarchical self-assembly," Soft Matter 12, 9633-9640 (2016).

${ }^{8}$ S. N. Fejer and D. J. Wales, "Design of a kagome lattice from soft anisotropic particles," Soft Matter 11, 6663-6668 (2015).

${ }^{9}$ G. van Anders, D. Klotsa, A. S. Karas, P. M. Dodd, and S. C. Glotzer, "Digital alchemy for materials design: Colloids and beyond," ACS Nano 9 , 9542-9553 (2015).

${ }^{10}$ C. X. Du, G. van Anders, R. S. Newman, and S. C. Glotzer, "Shape-driven solid-solid transitions in colloids," Proc. Natl. Acad. Sci. USA 114, E3892E3899 (2017).

${ }^{11}$ R. K. Cersonsky, G. van Anders, P. M. Dodd, and S. C. Glotzer, "Relevance of packing to colloidal self-assembly," Proc. Natl. Acad. Sci. USA (2018).

${ }^{12}$ N. Metropolis, A. W. Rosenbluth, N. M. Rosenbluth, A. H. Teller, and

E. Teller, "Equation of state calculations by fast computing machines," J. Chem. Phys. 21, 1087-1092 (1953).

${ }^{13}$ J. Proctor, G. van Anders, and S. C. Glotzer, "Digital alchemy applied to molecular dynamics," in APS Meeting Abstracts (2019).

${ }^{14}$ P. Zhou, J. Proctor, J. Dshemuchadse, G. van Anders, and S. C. Glotzer, "Inverse design for self-assembly of materials with targeted mechanical properties," in APS Meeting Abstracts (2019).

${ }^{15}$ Y. Geng, G. van Anders, P. M. Dodd, J. Dshemuchadse, and S. C. Glotzer, "Engineering entropy for the inverse design of colloidal crystals from hard shapes," Science Advances 5 (2019).

${ }^{16} \mathrm{M}$. Mihalkovič and C. L. Henley, "Empirical oscillating potentials for alloys from ab initio fits and the prediction of quasicrystal-related structures in the Al-Cu-Sc system," Phys. Rev. B 85, 092102 (2012).

${ }^{17}$ M. Engel, P. F. Damasceno, C. L. Phillips, and S. C. Glotzer, "Computational self-assembly of a one-component icosahedral quasicrystal," Nat. Mater. 14, 109-116 (2015).

${ }^{18}$ M. Hoare and P. Pal, "Physical cluster mechanics: Statics and energy surfaces for monatomic systems," Advances in Physics 20, 161-196 (1971).

${ }^{19} \mathrm{~S}$. D. Stoddard and J. Ford, "Numerical experiments on the stochastic behavior of a Lennard-Jones gas system,” Phys. Rev. A 8, 1504-1512 (1973).

${ }^{20}$ J. A. Anderson, C. D. Lorenz, and A. Travesset, "General purpose molecular dynamics simulations fully implemented on graphics processing units," J. Comput. Phys. 227, 5342-5359 (2008).

${ }^{21}$ J. Glaser, T. D. Nguyen, J. A. Anderson, P. Liu, F. Spiga, J. A. Millan, D. C. Morse, and S. C. Glotzer, "Strong scaling of general-purpose molecular dynamics simulations on gpus," Comput. Phys. Commun. 192, 97-107 (2015).

${ }^{22} \mathrm{Z}$. Li and H. A. Scheraga, "Monte Carlo-minimization approach to the multiple-minima problem in protein folding," Proc. Natl. Acad. Sci. USA 84, 6611-6615 (1987).

${ }^{23} \mathrm{Z}$. Li and H. A. Scheraga, "Structure and free energy of complex thermodynamic systems," J. Mol. Struc-Theochem 179, 333 (1988). 
${ }^{24}$ D. J. Wales and J. P. K. Doye, "Global optimization by basin-hopping and the lowest energy structures of Lennard-Jones clusters containing up to 110 atoms," J. Phys. Chem. A 101, 5111-5116 (1997).

${ }^{25}$ D. J. Wales, "GMIN: A program for basin-hopping global optimisation," (http://www-wales.ch.cam.ac.uk/software.html).

${ }^{26}$ J. Nocedal, "Updating quasi-Newton matrices with limited storage," Math. Comput. 35, 773-782 (1980).

${ }^{27}$ S. A. Trygubenko and D. J. Wales, "A doubly nudged elastic band method for finding transition states," J. Chem. Phys. 120, 2082-2094 (2004).

${ }^{28}$ S. A. Trygubenko and D. J. Wales, "Erratum: A doubly nudged elastic band method for finding transition states [j. chem. phys. 120, 2082 (2004)], J. Chem. Phys. 120, 7820-7820 (2004).

${ }^{29}$ D. Sheppard, R. Terrell, and G. Henkelman, "Optimization methods for finding minimum energy paths,” J. Chem. Phys. 128, 134106 (2008).

${ }^{30} \mathrm{G}$. Mills and H. Jónsson, "Quantum and thermal effects in $\mathrm{H}_{2}$ dissociative adsorption: Evaluation of free energy barriers in multidimensional quantum systems," Phys. Rev. Lett. 72, 1124-1127 (1994).

${ }^{31}$ G. Mills, H. Jónsson, and G. K. Schenter, "Reversible work transition state theory: application to dissociative adsorption of hydrogen," Surf. Sci. 324, 305-337 (1995).

${ }^{32}$ H. Jónsson, G. Mills, and K. W. Jacobsen, Classical and quantum dynamics in condensed phase simulations (World Scientific, Singapore, 1998).

${ }^{33}$ G. Henkelman, B. P. Uberuaga, and H. Jónsson, "A climbing image nudged elastic band method for finding saddle points and minimum energy paths," J. Chem. Phys. 113, 9901-9904 (2000).

${ }^{34} \mathrm{G}$. Henkelman and H. Jónsson, "Improved tangent estimate in the nudged elastic band method for finding minimum energy paths and saddle points," The Journal of Chemical Physics 113, 9978-9985 (2000).

${ }^{35}$ L. J. Munro and D. J. Wales, "Defect migration in crystalline silicon," Phys. Rev. B 59, 3969-3980 (1999)

${ }^{36}$ D. J. Wales, "OPTIM: A program for optimizing geometries and calculating reaction pathways," (http://www-wales.ch.cam.ac.uk/software.html).

${ }^{37}$ J. N. Murrell and K. J. Laidler, "Symmetries of activated complexes," Trans. Faraday Soc. 64, 371-377 (1968).

${ }^{38}$ C. J. Cerjan and W. H. Miller, "On finding transition states," J. Chem. Phys. 75, 2800 (1981).

${ }^{39}$ D. J. Wales, "PATHSAMPLE: A program for generating connected stationary point databases and extracting global kinetics," (http://wwwwales.ch.cam.ac.uk/software.html).

${ }^{40}$ D. J. Wales, J. P. K. Doye, M. A. Miller, P. N. Mortenson, and T. R. Walsh, "Energy landscapes: From clusters to biomolecules," Adv. Chem. Phys. 115, 1-111 (2000).

${ }^{41}$ Y. Kumeda, D. J. Wales, and L. J. Munro, "Transition states and rearrangement mechanisms from hybrid eigenvector-following and density functional theory: Application to $\mathrm{C}_{10} \mathrm{H}_{10}$ and defect migration in crystalline silicon," Chem. Phys. Lett. 341, 185-194 (2001).

${ }^{42}$ D. J. Wales, "Exploring energy landscapes," Annu. Rev. Phys. Chem. 69, 401-425 (2018)

${ }^{43}$ A. A. Hagberg, D. A. Schult, and P. J. Swart, "Exploring network structure, dynamics, and function using networkx," in Proceedings of the 7th Python in Science Conference (SciPy2008), edited by G. Varoquaux, T. Vaught, and J. Millman (Pasadena, CA USA, 2008) pp. 11-15.

${ }^{44}$ D. J. McGinty, "Vapor phase homogeneous nucleation and the thermodynamic properties of small clusters of argon atoms," J. Chem. Phys. 55, 580588 (1971).

${ }^{45}$ J. J. Burton, "Vibrational frequencies and entropies of small clusters of atoms," J. Chem. Phys. 56, 3133-3138 (1972).

${ }^{46}$ M. R. Hoare, "Structure and dynamics of simple microclusters," Adv. Chem. Phys. 40, 49-135 (1979).

${ }^{47}$ F. H. Stillinger and T. A. Weber, "Packing structures and transitions in liquids and solids," Science 225, 983-989 (1984).

${ }^{48}$ G. Franke, E. R. Hilf, and P. Borrmann, "The structure of small clusters: multiple normal modes model,’ J. Chem. Phys. 98, 3496-3502 (1993).

${ }^{49}$ D. J. Wales, "Coexistence in small inert-gas clusters," Mol. Phys. 78, 151171 (1993).

${ }^{50}$ B. Strodel and D. J. Wales, "Free energy surfaces from an extended harmonic superposition approach and kinetics for alanine dipeptide." Chem. Phys. Lett. 466, 105-115 (2008).

${ }^{51}$ F. G. Amar and R. S. Berry, "The onset of non-rigid dynamics and the melting transition in $\mathrm{Ar}_{7}$," J. Chem. Phys. 85, 5943 (1986).

${ }^{52}$ D. J. Wales, "Decoding heat capacity features from the energy landscape," Phys. Rev. E 95, 030105 (2017).

${ }^{53}$ A. Banerjee and N. P. Adams, "On coordinate transformations in steepest descent path and stationary point locations," Int. J. Quant. Chem. 43, 855871 (1992).

${ }^{54}$ J. D. Bryangelson, J. N. Onuchic, N. D. Socci, and P. G. Wolynes, "Funnels, pathways, and the energy landscape of protein folding: A synthesis," Proteins: Struct., Funct., Genet. 21, 167-195 (1995).

55 J. N. Onuchic, Z. Luthey-Schulten, and P. G. Wolynes, "Theory of protein folding: The energy landscape perspective," Annu. Rev. Phys. Chem. 48, 545-600 (1997)

${ }^{56}$ V. K. de Souza, J. D. Stevenson, S. P. Niblett, J. D. Farrell, and D. J. Wales, "Defining and quantifying frustration in the energy landscape: Applications to atomic and molecular clusters, biomolecules, jammed and glassy systems," J. Chem. Phys. 146, 124103 (2017).

${ }^{57}$ D. J. Wales and J. P. K. Doye, "Stationary points and dynamics in highdimensional systems," J. Chem. Phys. 119, 12409-12416 (2003).

${ }^{58}$ J. W. R. Morgan and D. J. Wales, "Energy landscapes of planar colloidal clusters," Nanoscale 6, 10717-10726 (2014).

${ }^{59}$ O. M. Becker and M. Karplus, "The topology of multidimensional potential energy surfaces: theory and application to peptide structure and kinetics," J. Chem. Phys. 106, 1495-1517 (1997).

${ }^{60}$ D. J. Wales, M. A. Miller, and T. A. Walsh, "Archetypal energy landscapes," Nature 394, 758-760 (1998).

${ }^{61}$ J. Patel and M. Cohen, "Criterion for the action of applied stress in the martensitic transformation," Acta Metall. 1, 531-538 (1953).

${ }^{62} \mathrm{P}$. M. Morse, "Diatomic molecules according to the wave mechanics. ii. vibrational levels," Phys. Rev. 34, 57-64 (1929). 